\title{
10 Referencias bibliográficas
}

Agencia EFE, Manual de español urgente, Madrid, Cátedra, 1992.

Alarcos Llorach, Emilio, Estudios de gramática funcional española, Madrid, Gredos, 1973.

Alarcos Llorach, Emilio, Gramática de la lengua española, Madrid, Espasa-Calpe, 1994.

Alcaraz Varó, Enrique/Martínez Linares, M. ${ }^{a}$ Antonia, Diccionario de lingüística moderna, Barcelona, Ariel, 1997.

Alcina, Juan/Blecua, Juan Manuel, Gramática española, Barcelona, Ariel, 1991.

Almeida, Manuel, (De)queísmo y variación sociolingüística en una comunidad urbana canaria, Revista de filología 27 (2009), 9-30 (= 2009a).

Almeida, Manuel, «Priming» y efectos mecánicos en la variación lingüística: el «(de)queísmo» en una comunidad canaria, Boletín de filología de la Universidad de Chile 44:1 (2009), 11-37 (= 2009b).

Almeida, Manuel, El (de)queísmo en una comunidad urbana canaria: norma social y actitudes, Revista internacional de lingüística iberoamericana 16 (2010), 29-47.

Alonso del Río, José, Gramática española, Madrid, Giner, 1963.

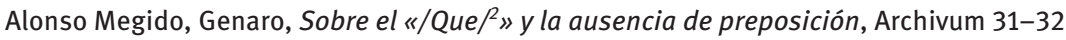
(1981-1982), 65-84.

Ambruzzi, Lucio, Gramática spagnola, Torino, Società Editrice Internazionale, 1955.

Amorós, Carla, El queísmo a la luz de las gramáticas españolas contemporáneas, Interlingüística 18 (2009), 124-134.

Ariza, Manuel, Notas sobre el español de América (las cartas privadas de viajeros a Indias), Cauce 14-15 (1992), 25-36.

Arjona, Marina, Anomalías en el uso de la preposición «de» en el español de México, Anuario de letras 16 (1978), 67-90.

Arjona, Marina, Usos anómalos de la preposición «de» en el habla popular mexicana, Anuario de letras 17 (1979), 167-184.

Auer, Anita/Peersman, Catharina/Pickl, Simon/Rutten, Gijsbert/Vosters, Rik, Historical sociolinguistics: the field and its future, Journal of historical sociolinguistics 1:1 (2015), 1-12.

Badia, Antoni M., Gramática catalana, Madrid, Gredos, 1994.

Bentivoglio, Paola, El dequeísmo en Venezuela: ¿un caso de ultracorrección?, Boletín de filología de la Universidad de Chile 31. Homenaje a Ambrosio Rabanales (1980-1981), 705-719.

Bentivoglio, Paola/D’Introno, Francesco, Análisis sociolingüístico del dequeísmo en el habla de Caracas, Boletín de la Academia Puertorriqueña de la Lengua Española 6:1 (1977), 58-82.

Bentivoglio, Paola/Galué, Dexy, Ausencia y presencia de la preposición «de» ante cláusulas encabezadas por "que» en el español de Caracas: un análisis variacionista, Boletín de filología de la Universidad de Chile 37:1 (1998-1999), 139-159.

Bentivoglio, Paola/Sedano, Mercedes, Morphosyntactic variation in Spanish-speaking Latin America, in: Díaz-Campos, Manuel (ed.), The handbook of Hispanic sociolinguistics, Oxford, Wiley/Blackwell, 2011, 168-186.

Bergs, Alexander, Social network analysis and historical sociolinguistics, Berlin/New York, Mouton de Gruyter, 2005.

Bergs, Alexander, The uniformitarian principle and the risk of anachronisms in language and social history, in: Hernández-Campoy, Juan Manuel/Conde-Silvestre, Juan Camilo (edd.), The handbook of historical sociolinguistics, Malden, MA, Wiley/Blackwell, 2012, 80-98.

Ә Open Access. () 2022 José Luis Blas Arroyo and Mónica Velando Casanova, published by De Gruyter. (c) BY-NC-ND This work is licensed under the Creative Commons Attribution-NonCommercial-NoDerivatives 4.0 International License.

https://doi.org/10.1515/9783110766851-010 
Blas Arroyo, José Luis, Sociolingüística del español, Madrid, Cátedra, 2005.

Blas Arroyo, José Luis, The rise and fall of a change from below in Early Modern Spanish. The periphrasis « deber de + infinitive» in texts of linguistic immediacy, Journal of historical linguistics 6:1 (2016), 1-31.

Blas Arroyo, José Luis, ¿Identidades lingüísticas invariables?: trayectorias idiolectales ante la variación y el cambio lingüístico en el español clásico, Revista internacional de lingüística iberoamericana (RILI) 15:2 (2017), 117-144.

Blas Arroyo, José Luis/Porcar, Margarita, De la función a la forma: la influencia del contexto variable en la selección de las perífrasis modales de infinitivo en el primer español clásico, Anuario de lingüística hispánica 30 (2014), 9-49.

Blas Arroyo, José Luis/Porcar, Margarita, «Tiene tanto temor a la mar que creo no lo hará». Variación en la sintaxis de las completivas en los Siglos de Oro, in: Arnal Purroy, María Luisa/Enguita, José María/Castañer, Rosa María/Lagüéns, Vicente/Martín Zorraquino, María Antonia (edd.), Actas del X Congreso Internacional de Historia de la lengua española, Zaragoza, Universidad de Zaragoza/Institución Fernando el Católico, 2018, 532-548.

Blas Arroyo, José Luis/Schulte, Kim, Competing modal periphrases in Spanish between the 16th and the 18th century. A diachronic variationist approach, Diachronica 34:1 (2017), 1-39.

Blas Arroyo, José Luis/Vellón, Javier, On the trail of grammaticalisation in progress: has «el que» become a compound relative pronoun in the history of Spanish prepositional relative clauses?, Probus. International journal of Romance linguistics 30:1 (2018), 1-45.

Blas Arroyo, José Luis/Porcar, Margarita/Velando, Mónica/Vellón, Javier, Sociolingüística histórica del español. Tras las huellas de la variación y el cambio lingüístico a través de textos de inmediatez comunicativa, Madrid/Frankfurt, Iberoamericana/Vervuert, 2019.

Bogard, Sergio/Company, Concepción, Estructura y evolución de las oraciones completivas de sustantivo en el español, Romance philology 43:2 (1989), 258-273.

Boretti de Macchia, Susana, (De)queísmo en el habla culta de Rosario, Anuario de lingüística hispánica 5 (1989), 27-48.

Boretti de Macchia, Susana, (Des)uso preposicional en el habla culta: queísmo y dequeísmo, in: Donni de Mirande, Nélida/Boretti de Macchia, Susana/Ferrer de Gregoret, M. ${ }^{a}$ Cristina/ Sánchez Lanza, Carmen (edd.), Variación lingüística en el español de Rosario, Rosario, Universidad Nacional de Rosario, 1991, 115-123.

Brucart, José M. a , La estructura del sintagma nominal: las oraciones de relativo, in: Bosque, Ignacio/Demonte, Violeta (edd.), Gramática descriptiva de la lengua española, vol. 2, Madrid, Espasa-Calpe, 1999, 395-522.

Burke, Peter, History and social theory, Cambridge, Polity Press, 1992.

Bybee, Joan L., Morphology. A study between the relation of meaning and form, Amsterdam, John Benjamins, 1985.

Bybee, Joan L., From usage to grammar. The mind's response to repetition, Language 82 (2006), 711-733.

Bybee, Joan L./Hopper, Paul (edd.), Frequency and the emergence of linguistic structure, Amsterdam, John Benjamins, 2001.

Bybee, Joan L./Thompson, Sandra, Three frequency effects in syntax, in: Juge, Matthew L./ Moxley, Jeri L. (edd.), Proceedings of the 23rd annual meeting of the Berkeley Linguistics Society. General session and parasession on pragmatics and grammatical structure, Berkeley, Berkeley Linguistics Society, 2000, 378-388.

Calero Calero, Francisco, Sintaxis latina, Madrid, UNED, 2003. 
Cano Aguilar, Rafael, El régimen de las oraciones completivas en el español clásico, Madrid, Gredos, 1985.

Cano Aguilar, Rafael, Lenguaje «espontáneo» y retórica epistolar en cartas de emigrantes españoles a Indias, in: Kotschi, Thomas/Oesterreicher, Wulf/Zimmermann, Klaus (edd.), El español hablado y la cultura oral en España e Hispanoamérica, Madrid/Frankfurt am Main, Iberoamericana/Vervuert, 1996, 375-404.

Carbonero, Pedro, Sobre ciertas construcciones de relativo en el habla urbana de Sevilla, in: Rodríguez Izquierdo, Fernando (ed.), Sociolingüística andaluza, vol. 3, Sevilla, Publicaciones de la Universidad de Sevilla, 1985, 65-85.

Carbonero, Pedro, Queísmo y dequeísmo en el habla culta de Sevilla: análisis contrastado con otras hablas peninsulares y americanas, in: Luna, Elisabeth (ed.), Scripta philologica in honorem Juan M. Lope Blanch, vol. 2, México, Universidad Nacional Autónoma de México, 1991, 43-63.

Cecchini, Mario, Manual de sintaxis española, Napoli, Liguori, 1968.

Cestero, Ana María/Molina Martos, Isabel/Paredes, Florentino, La lengua hablada en Madrid. Corpus PRESEEA-Madrid (distrito de Salamanca) (edición en CD-ROM), Alcalá de Henares, Universidad de Alcalá, 2012.

Company Company, Concepción, La frase sustantiva en el español medieval. Cuatro cambios sintácticos, México, Universidad Nacional Autónoma de México, 1991.

Company Company, Concepción/Bogard, Sergio, Las oraciones completivas de nombre sin preposición en el español de México: una perspectiva diacrónica, in: Moreno de Alba, José G. (ed.), Actas del II Congreso Internacional sobre el español de América, México, Universidad Nacional Autónoma de México, 1986, 243-248.

Conde-Silvestre, Juan Camilo, Sociolingüística histórica, Madrid, Gredos, 2007.

Conde-Silvestre, Juan Camilo/Hernández-Campoy, Juan Manuel, A sociolinguistic approach to the diffusion of Chancery written practices in late fifteenth-century private correspondence, Neuphilologische Mitteilungen 105 (2004), 133-152.

Conde-Silvestre, Juan Camilo/Hernández-Campoy, Juan Manuel, Sociolinguistic and geolinguistic approaches to the historical diffusion of linguistic innovations. Incipient standardisation in Late Middle English, International journal of English studies 5:1 (2005), 101-134.

Cornillie, Bert/Delbecque, Nicole, Speaker commitment. Back to the speaker evidence from spanish alternations, Belgian journal of linguistics 22 (2008), 37-62.

Corominas, Joan/Pascual, José A., Diccionario crítico etimológico castellano e hispánico, 6 vol., Madrid, Gredos, 1980-1990.

Cortés Rodríguez, Luis, Usos anómalos del relativo en el español hablado, Revista española de lingüística 20:2 (1990), 431-446.

Coste, Jean/Redondo, Augustin, Syntaxe de l'espagnol moderne, Madrid, SEDES, 1965.

Criado del Val, Manuel, Gramática española y comentario de textos, Madrid, Saeta, 1958.

Croft, William, Explaining language change. An evolutionary approach, Harlow, Longman, 2000.

Cuartas, Augusto/Santamaría, Andrés, Diccionario de incorrecciones y particularidades del lenguaje, Madrid, Paraninfo, 1967.

Culpeper, Jonathan/Kytö, Merja, Early modern English dialogues. Spoken interaction as writing, Cambridge, Cambridge University Press, 2010.

Davies, Mark, Corpus del español, 2002, http://www.corpusdelespanol.org. [último acceso: 11.01.2021] 
Del Moral, Carlos G., Grammaticalization of Spanish «de». Reanalysis of «(de)queísmo» in Southern Cone dialects, Doctoral thesis, University of Illinois at UrbanaChampaign, 2004.

Del Valle Rodás, Juana, Para una lingüística interpretativa. (De)queísmo en el habla de Salta (N. O. argentino), Anuario de lingüística hispánica 12-13 (1996-1997), 797-818.

DeMello, George, El dequeísmo en el español hablado contemporáneo. ¿Un caso de independencia semántica?, Hispanic linguistics 6:7 (1995), 117-152.

Demonte, Violeta, La esquiva norma del español. Sus fusiones y relaciones con la variación y el estándar, in: Álvarez, Rosario/Monteagudo, Henrique (edd.), Norma lingüística e variación, Santiago de Compostela, Instituto da Lingua Galega/Consello da Cultura Galega, 2004, 13-30.

Demonte, Violeta/Fernández Soriano, Olga, Features in comp and syntactic variation. The case of «(de)queísmo» in Spanish, Lingua 115:8 (2005), 1063-1082.

Dietrick, Deborah, De (queísmo) y cuestiones afines en el habla culta de San Juan de Puerto Rico, in: El español de América. Actas del IV congreso internacional del español de América, Santiago de Chile, Mar del Plata, 1992, 665-677.

Dossena, Marina/Tieken-Boon van Ostade, Ingrid (edd.), Studies in late modern English correspondence. Methodology and data, Bern, Peter Lang, 2008.

Echeverría, Carlos, Queísmo en el español de Chile. Del habla culta al habla presidencial, Comunicaciones en humanidades 5 (2016), 234-243.

Eckert, Penelope, Linguistic variation as social practice, Oxford, Blackwell, 2000.

El País, Libro de estilo, Madrid, El País, 1990.

Elliott, John, La España imperial, Barcelona, Vicens-Vives, 1998.

Fernández Álvarez, Manuel, Sombras y luces en la España imperial, Barcelona, Espasa, 2004.

Fernández Ordóñez, Inés, Transmisión manuscrita y transformación «discursiva» de los textos, in: Bustos Tovar, José Jesús/Girón Alconchel, José Luis (edd.), Actas del VI congreso de historia de la lengua española, Madrid, Arco/Libros, 2006, 3033-3045.

Fernández Ordóñez, Inés, El norte peninsular y su papel en la historia de la lengua española, in: Gómez Seibane, Sara/Sinner, Carsten (edd.), Estudios sobre tiempo y espacio en el español norteño, San Millán de la Cogolla (La Rioja), Cilengua, 2011, 23-68.

Fontanella de Weinberg, María B., El español de América, Madrid, Maphre, 1992.

Galué, Dexy, «Me acuerdo que...»: los verbos pronominales y el queísmo, in: Knauer, Gabriele/ Bellosta von Colbe, Valeriano (edd.), Variación sintáctica en español, Tübingen, Niemeyer, 2005, 221-232.

García, Erica, El fenómeno (de)queísmo desde una perspectiva dinámica del uso de la lengua, in: Moreno de Alba, José G. (ed.), Actas del II congreso internacional sobre el español de América, México, Universidad Nacional Autónoma de México, 1986, 46-65.

García Mouton, Pilar, Tratamientos en las cartas de Indias, in: Forastieri, Eduardo/Cardona, Julia/López Morales, Humberto/Morales, Amparo (edd.), Estudios de lingüística hispánica. Homenaje a María Vaquero, San Juan, Editorial de la Universidad de Puerto Rico, 1999, 263-276.

García Yebra, Valentín, Claudicación en el uso de las preposiciones, Madrid, Gredos, 1990.

Girón Alconchel, José Luis, Cambios gramaticales en los Siglos de Oro, in: Cano Aguilar, Rafael (coord.), Historia de la lengua española, Barcelona, Ariel, 2004, 859-893.

Givón, Talmy, On understanding grammar, New York, Academic Press, 1979.

Gómez Devís, María Begoña, El queísmo: causas y actitudes sociolingüísticas, in: Briz, Antonio/ Gómez Molina, José Ramón/Martínez Alcalde, M. ${ }^{a}$ José (edd.), Pragmática y gramática 
del español hablado. Actas del II Simposio sobre análisis del discurso oral, València/ Zaragoza, Universitat de València/Pórtico Libros, 1996, 311-316.

Gómez Molina, José Ramón, Dequeísmo y queísmo en el español hablado de Valencia, in: Briz, Antonio/Gómez Molina, José Ramón/Martínez Alcalde, M. ${ }^{a}$ José (edd.), Pragmática y gramática del español hablado. Actas del Il simposio sobre análisis del discurso oral, València/Zaragoza, Universitat de València/Pórtico Libros, 1996, 75-89.

Gómez Molina, José Ramón, Las construcciones «de/Ø + que + verbo en forma personal», in: Gómez Molina, José R. (ed.), El español de Valencia. Estudio sociolingüístico, Bern, Peter Lang, 2013, 183-222.

Gómez Molina, José Ramón/Gómez Devís, María Begoña, Dequeísmo y queísmo en el español hablado de Valencia: factores lingüísticos y sociales, Anuario de lingüística hispánica 11 (1995), 193-220.

Gómez Torrego, Leonardo, La variación en las subordinadas sustantivas: dequeísmo y queísmo, in: Bosque, Ignacio/Demonte, Violeta (edd.), Gramática descriptiva de la lengua española, vol. 2, Madrid, Espasa-Calpe, 1999, 2105-2148.

Gómez Torrego, Leonardo, Hablar y escribir correctamente. Gramática normativa del español actual, Madrid, Arco/Libros, 2006.

Granda, Germán de, El proceso de koineización en el período inicial de desarrollo del español de América, in: Lüdtke, Jens (ed.), El español de América en el siglo XVI. Actas del simposio del Instituto Ibero-Americano de Berlín, Frankfurt/Madrid, Vervuert/Iberoamericana, 1994, 87-108.

Guirado, Krístel, (De)queísmo: uso deíctico y distribución social en el habla de Caracas, Caracas, Universidad Central de Venezuela, 2009.

Gutiérrez Araus, M. ${ }^{a}$ Luz, Sobre la elisión de preposición ante «que» relativo, Lingüística española actual 7:1 (1985), 15-36.

Gutiérrez Araus, M. ${ }^{a}$ Luz, La omisión de preposición ante relativo «que» en el español de Puerto Rico, in: Moreno de Alba, José G. (ed.), Actas del Il congreso internacional sobre el español de América, México, Universidad Nacional Autónoma de México, 1986, 407-417.

Hebda, Anna, Phonological variables, in: Hernández-Campoy, Juan Manuel/Conde-Silvestre, Juan Camilo (edd.), The handbook of historical sociolinguistics, Malden, MA, Wiley/ Blackwell, 2012, 237-251.

Herdeiro, Ana/Barbosa, Pilar, o fenómeno do queísmo no falar bracarense: um estudo sociolinguístico, Diacrítica 29:1 (2015), 327-351.

Herman, József, El latín vulgar, Barcelona, Ariel, 2001.

Hernández, César, Gramática funcional del español, Madrid, Gredos, 1996.

Hernández-Campoy, Juan Manuel/Schilling-Estes, Natalie, The application of the quantitative paradigm to historical sociolinguistics. Problems with the generalizability principle, in: Hernández-Campoy, Juan Manuel/Conde-Silvestre, Juan Camilo (edd.), The handbook of historical sociolinguistics, Malden, MA, Wiley/Blackwell, 2012, 63-79.

Herrera Santana, Juana, La ausencia de preposición ante «que»: ¿un paso hacia su gramaticalización?, Revista de filología de la Universidad de La Laguna 15 (1997), 109-117.

Herrero Ruiz de Loizaga, Francisco Javier, Sintaxis histórica de la oración compuesta, Madrid, Gredos, 2005.

Herrero Ruiz de Loizaga, Francisco Javier, La conjunción «que». La complejización del sistema de subordinación, in: Company Company, Concepción (ed.), Sintaxis histórica de la lengua española. Tercera parte: preposiciones, adverbios y conjunciones. Relaciones interora- 
cionales, tomo 2, México, Universidad Nacional Autónoma de México/Fondo de Cultura Económica, 2014, 2789-2970.

Johnson, Daniel Ezra, Getting off the GoldVarb standard. Introducing Rbrul for mixed-effects variable rule analysis, Language and linguistics compass 3:1 (2009), 359-383.

Kanwit, Matthew, The role of discourse topic in evidentiality marking. Variable "(de)queísmo» in Caracas, eHumanista/IVITRA 8 (2015), 446-470.

Kany, Charles E., Sintaxis hispanoamericana, Madrid, Gredos, 1969.

Keniston, Hayward, The syntax of Castilian prose. The sixteenth century, Chicago, The University of Chicago Press, 1937.

Kerswill, Paul, Koineization, in: Chambers, Jack/Schilling, Natalie (edd.), The handbook of language variation and change, Malden, MA, Wiley/Blackwell, 2013, 519-535.

Kielkiewicz-Janowiak, Agnieszka, Class, age, and gender-based patterns, in: Hernández-Campoy, Juan Manuel/Conde-Silvestre, Juan Camilo (edd.), The handbook of historical sociolinguistics, Malden, MA, Wiley/Blackwell, 2012, 307-330.

King, Larry D., The semantic structure of Spanish: meaning and grammatical form, Amsterdam, Holanda/Philadelphia, PA, John Benjamins, 1992.

Koch, Peter/Oesterreicher, Wulf, Sprache der Nähe - Sprache der Distanz. Mundlichkeit und

Schriftlichkeit im Spannungsfeld von Sprachtheorie und Sprachgeschichte, Romanis-

tisches Jahrbuch 36 (1985), 15-43.

Labov, William, Sociolinguistics patterns, Philadelphia, Pennsylvania UP, 1972.

Labov, William, Principles of linguistic change, vol. 2: Social factors, Oxford, Blackwell, 2001.

Langacker, Ronald, Foundations of cognitive grammar, vol. 1: Theoretical prerequisites,

Stanford, Stanford University Press, 1987.

Lapesa, Rafael, Historia de la lengua española, Madrid, Gredos, 1980.

Lavandera, Beatriz R., El cambio de modo como estrategia de discurso, in: Bosque, Ignacio

(ed.), Indicativo y subjuntivo, Madrid, Taurus, 1990, 330-357.

Lázaro Carreter, Fernando, El dequeísmo, in: El Comercio (Lima), 2 de julio de 1981.

Lenz, Rodolfo, La oración y sus partes, Madrid, Centro de Estudios Históricos, 1935.

Leonetti, Manuel, La subordinación sustantiva: las subordinadas enunciativas en los complementos nominales, in: Bosque, Ignacio/Demonte, Violeta (edd.), Gramática descriptiva de la lengua española, vol. 2, Madrid, Espasa-Calpe, 1999, 2083-2104.

Llorente Maldonado, Antonio, Consideraciones sobre el español actual, Anuario de letras 18 (1980), 5-61.

Lope Blanch, Juan M., Peculiaridades sintácticas de los relativos en el habla culta de Madrid, in: Borrego, Julio/Gómez Asencio, José J./Santos, Luis (edd.), Philologica. Homenaje a Antonio Llorente, Salamanca, Universidad de Salamanca, 1989, 79-85.

Lope Blanch, Juan M., Nexos temporales en las «Cartas» de Hernán Cortés, Lexis 20:1-2 (1996), 405-420.

Lope Blanch, Juan M., Los relativos en problemas, Lexis 25:1-2 (2001), 159-172.

López Morales, Humberto, Sociolingüística, Madrid, Gredos, 2004.

Maldonado, Concepción (ed.), Clave. Diccionario de uso del español actual, Madrid, SM, 1996.

Malkiel, Yakov, A tentative typology of Romance historical grammars, in: Malkiel, Yakov, Essays on linguistic themes, Oxford, Oxford University Press, 1968, 71-164.

Marcos Marín, Francisco, Aproximación a la gramática española, Madrid, Cincel, 1984.

Marcos Marín, Francisco/Satorre Grau, Javier, Gramática española, Barcelona, Letras Universitarias/Síntesis, 1998. 
Martínez Amador, Emilio, Diccionario gramatical y de dudas del idioma, Barcelona, Ramón Sopena, 1974.

Martínez Sequeira, Ana T., El dequeísmo en el español de Costa Rica. Un análisis semántico-pragmático, Tesis doctoral, University of Southern California, 2000.

McLauchlan, Jessica, Dequeísmo y queísmo en el habla culta de Lima, Lexis 6:1 (1982), 11-55.

Menéndez Pidal, Ramón, Cantar de Mio Cid (vocabulario, texto y gramática), 2 vol., Madrid, Espasa-Calpe, 1969.

Millán, Alberto, Queísmo y dequeísmo en los alumnos de la Facultad de Filología de la Universidad de Sevilla, Cauce. Revista de filología y su didáctica 22-23 (1999-2000), 495-518.

Milroy, Lesley, Language and social networks, Oxford, Blackwell, 1987.

Mollica, María Cecilia, Processing and morpho-semantic effects in complementation in Brazilian Portuguese, Language variation and change 3 (1991), 265-274.

Moreno de Alba, José G., Sintagmas completivos del nombre: complementos adnominales y oraciones subordinadas completivas del nombre, in: Company Company, Concepción (ed.), Sintaxis histórica de la lengua española. Segunda parte: la frase nominal, México, Universidad Nacional Autónoma de México/Fondo de Cultura Económica, 2009, 1321-1409.

Moreno Fernández, Francisco, Principios de sociolingüística y sociología del lenguaje, Barcelona, Ariel, 1998.

Moreno Fernández, Francisco, Historia social de las lenguas de España, Barcelona, Ariel, 2005.

Moreno Fernández, Francisco/Cestero Mancera, Ana María/Molina Martos, Isabel/Paredes, Florentino, La lengua hablada en Alcalá de Henares. Corpus PRESEEA-Alcalá (edición en CD-ROM), Alcalá de Henares, Universidad de Alcalá, 2002-2007.

Mozas, Antonio Benito, Gramática práctica, Madrid, Edaf, 1992.

Náñez, Emilio, Sobre dequeísmo, Revista de filología románica 2 (1984), 239-248.

Nevalainen, Terttu, Social stratification, in: Nevalainen, Terttu/Raumolin-Brunberg, Helena (edd.), Sociolinguistics and language history. Studies based on the Corpus of early English correspondence, Amsterdam/Atlanta, GA, Rodopi, 1996, 57-76.

Nevalainen, Terttu, Making the best use of «bad» data: evidence for socio-linguistic variation in early modern English, Neuphilologische Mitteilungen 100:4 (1999), 499-533.

Nevalainen, Terttu/Raumolin-Brunberg, Helena (edd.), Sociolinguistics and language history. Studies based on the Corpus of early English correspondence, Amsterdam/Atlanta, GA, Rodopi, 1996.

Nevalainen, Terttu/Raumolin-Brunberg, Helena, Historical sociolinguistics, London, Longman, 2003. Nevalainen, Terttu/Raumolin-Brunberg, Helena/Mannila, Heikki, The diffusion of language change in real time: progressive and conservative individuals and the time-depth of change, Language variation and change 23:1 (2011), 1-43.

Nurmi, Arja/Nevala, Minna/Palander-Collin, Minna (edd.), The language of daily life in England (1400-1800), Amsterdam, John Benjamins, 2009.

Oesterreicher, Wulf, Lo hablado en lo escrito. Reflexiones metodológicas y aproximación a una tipología, in: Kotschi, Thomas/Oesterreicher, Wulf/Zimmermann, Klaus (edd.), El español hablado y la cultura oral en España e Hispanoamérica, Madrid/Frankfurt am Main, Iberoamericana/Vervuert, 1996, 317-340.

Oesterreicher, Wulf, Textos entre inmediatez y distancia comunicativas. El problema de lo hablado escrito en el Siglo de Oro, in: Cano Aguilar, Rafael (ed.), Historia de la lengua española, Barcelona, Ariel, 2004, 729-769. 
Okulska, Urszula, Performing the world of politics through the discourse of institutional correspondence in late middle and early modern England, in: Okulska, Urszula/Cap, Piotr (edd.), Perspectives in politics and discourse, Amsterdam, John Benjamins, 2010, 173-197.

Pahta, Päivi/Jucker, Andreas H. (edd.), Communicating early English manuscripts, Cambridge, Cambridge University Press, 2011.

Palander-Collin, Minna, Male and female styles in 17th century correspondence: I THINK, Language variation and change 11:2 (1999), 123-141.

Palander-Collin, Minna/Nevala, Minna (edd.), Letters and letter writing, European journal of English studies 9:1 (2005), 1-7.

Penny, Ralph, Variation and change in Spanish, Cambridge, Cambridge University Press, 2000. Pereira-Scherre, Maria M./Naro, Anthony J., The serial effect on internal and external variables, Language variation and change 4:1 (1992), 1-13.

Pérez Rioja, José A., Gramática de la lengua española, Madrid, Tecnos, 1953.

Pickering, Martin J./Ferreira, Victor S., Structural priming: a critical review, Psychological bulletin 134:3 (2008), 427-459.

Poplack, Shana, Grammaticalization and linguistic variation, in: Heine, Bernd/Narrog, Heiko (edd.), Handbook of grammaticalization, Oxford, Oxford University Press, 2011, 209-224.

Poplack, Shana/Dion, Nathalie, Prescription vs. praxis: the evolution of future temporal reference in French, Language 85:3 (2009), 557-587.

Pottier, Bernard, Gramática del español, Madrid, Alcalá, 1975.

Pountain, Christopher J., Preposición + «que» en español, Cuadernos de lingüística de El Colegio de México 2 (2014), 5-54.

Pountain, Christopher J., "Que»-deletion: the rise and fall of a syntactic fashion, in: Dubert, Francisco/Rei-Doval, Gabriel/Sousa, Xulio (edd.), En memoria de tanto miragre. Estudos dedicados ó profesor David Mackenzie, Santiago de Compostela, Universidade de Santiago de Compostela, 2015, 143-59.

Queen, Robin, Gender, sex, sexuality, and sexual identities, in: Chambers, Jack/Schilling, Natalie (edd.), The handbook of language variation and change, Malden, MA, Wiley/ Blackwell, 2013, 368-386.

Rabanales, Ambrosio, Queísmo y dequeísmo en el español de Chile, Onomázein 12:2 (2005), 23-53. Versión corregida y aumentada de la publicada en Tejera, María Josefina (ed.), Homenaje a Ángel Rosenblat en sus 70 años. Estudios filológicos y lingüísticos, Caracas, Instituto Pedagógico, 1974, 413-444.

RAE (Real Academia Española), Gramática de la lengua española. Nueva edición reformada, Madrid, Espasa-Calpe, 1931.

RAE (Real Academia Española), Esbozo de una nueva gramática de la lengua española, Madrid, Espasa-Calpe, 1973.

RAE (Real Academia Española)/ASALE (Asociación de Academias de la Lengua Española), Diccionario panhispánico de dudas, 2005, https://www.rae.es/dpd/. [último acceso: 02.01.2021]

RAE (Real Academia Española)/ASALE (Asociación de Academias de la Lengua Española), Nueva gramática de la lengua española, Madrid, Espasa, 2009.

Raible, Wolfgang, Knowing and believing - and syntax, in: Parret, Herman (ed.), On believing. De la croyance. Epistemological and semiotic approaches, Berlin, De Gruyter, 1983, 274-291.

Raumolin-Brunberg, Helena, Language change in adulthood. Historical letters as evidence, European journal of English studies 9:1 (2005), 37-51. 
Raumolin-Brunberg, Helena, Leaders of linguistic change in early modern England, in: Facchinetti, Roberta/Rissanen, Matti (edd.), Corpus-based studies of diachronic English, Bern, Peter Lang, 2006, 115-134.

Raumolin-Brunberg, Helena, Lifespan changes in the language of three early modern gentlemen, in: Nurmi, Arja/Nevala, Minna/Palander-Collin, Minna (edd.), The language of daily life in England (1400-1800), Amsterdam, John Benjamins, 2009, 165-196.

Rivera Mercado, María del Pilar, Queísmo y dequeísmo en la norma popular de la ciudad de San Juan, Tesis de maestría inédita, Universidad de Puerto Rico, Recinto de Río Piedras, 1998.

Rosemeyer, Malte/Schwenter, Scott A., Entrenchment and persistence in language change: the Spanish past subjunctive, Corpus linguistics and linguistic theory 15:1 (2019), 167-204.

Rutkowska, Hanna/Rössler, Paul, Orthographic variables, in: Hernández-Campoy, Juan Manuel/ Conde-Silvestre, Juan Camilo (edd.), The handbook of historical sociolinguistics, Malden, MA, Wiley/Blackwell, 2012, 213-235.

San Martín, Abelardo, Análisis sociolingüístico del queísmo en el español hablado en Santiago de Chile, Estudios filológicos 58 (2016), 207-228.

Sánchez-Prieto, Pedro, Cómo editar los textos medievales, Madrid, Arco/Libros, 1998.

Schmid, Hans-Jörg, Entrenchment, salience and basic levels, in: Geeraerts, Dirk/Cuyckens, Hubert (edd.), The Oxford handbook of cognitive linguistics, Oxford, Oxford University Press, 2012, 115-138.

Schneider, Edgar, Investigating variation and change in written documents, in: Chambers, Jack/Schilling, Natalie (edd.), The handbook of language variation and change, Malden, MA, Wiley/Blackwell, 2013, 57-81.

Schwenter, Scott A., Evidentiality in Spanish morphosyntax: a reanalysis of «(de)queísmo», in: Serrano, María José (ed.), Estudios de variación sintáctica, Madrid/Frankfurt am Main, Iberoamericana/Vervuert, 1999, 65-87.

Seco, Manuel, Diccionario de dudas y dificultades de la lengua española, Madrid, Espasa Calpe, 1989.

Seco, Manuel, Gramática esencial del español, Caracas, El Nacional, 2001.

Seco, Rafael, Manual de gramática española, Madrid, Aguilar, 1960.

Serradilla, Ana María, Sobre las primeras apariciones de construcciones preposicionales ante «que» completivo en español medieval. Factores determinantes, EPOS XI (1995), 147-163.

Serradilla, Ana María, Las formas analíticas de «entendimiento y lengua» ante «que» completivo en español medieval. Breves notas acerca de su evolución en la época clásica, Revista de filología románica 13 (1996), 13-38.

Serradilla, Ana María, El régimen de los verbos de entendimiento y lengua en español medieval, Madrid, Universidad Autónoma de Madrid, 1997.

Serradilla, Ana María, La distribución sociolingüística del quesuismo en los textos medievales y clásicos. Factores que propician su aparición, Estudios de lingüística del español 42 (2020), 11-38.

Serrano, María José, Gramática del discurso, Madrid, Akal, 2006.

Tagliamonte, Sali, Variationist sociolinguistics: change, observation, interpretation, Oxford, Wiley/Blackwell, 2012.

Tagliamonte, Sali A./Durham, Mercedes/Smith, Jennifer, Grammaticalization at an early stage. Future be going to in conservative British dialects, English language and linguistics 18:1 (2014), 75-108.

Tarr, Frederick Courtney, Prepositional complementary clauses in Spanish with special reference to the works of Pérez Galdós, Revue hispanique 56 (1922), 1-264. 
Torres-Cacoullos, Rena/Walker, James A., The present of the English future. Grammatical variation and collocations in discourse, Language 85 (2009), 321-354.

Tuten, Donald, Koineization in medieval Spanish, Berlin/New York, De Gruyter, 2003.

Ueda, Hiroto, Asimetría unidireccional de queísmo y dequeísmo. Aproximación a la realidad histórica y actual de la variación a través de estudios anteriores, corpus y encuestas, in: Spanish dialect syntax, Madrid, Universidad Complutense de Madrid, 2017, 1-57, https:// lecture.ecc.u-tokyo.ac.jp/ cueda/kenkyu/rekisi/de-queismo/dequeismo.pdf. [último acceso 02.01.2021].

Väänänen, Veikko, Introducción al latín vulgar, trad. por Manuel Carrión, Madrid, Gredos, 1967.

Van der Wal, Marijke J./Rutten, Gijsbert, Ego-documents in a historical-sociolinguistic perspective, in: Van der Wal, Marijke J./Rutten, Gijsbert (edd.), Touching the past. Studies in the historical sociolinguistics of ego-documents, Amsterdam/Philadelphia, John Benjamins, 2013, 1-18.

Wheeler, Max/Yates, Alan/Dols, Nicolau, Catalan. A comprehensive grammar, London/New York, Routledge, 1999. 